\title{
La culture corporelle
}

\section{Bernard-Xavier René}

\section{(2) OpenEdition}

Journals

Édition électronique

URL : http://journals.openedition.org/trema/2141

DOI : $10.4000 /$ trema. 2141

ISSN : 2107-0997

\section{Éditeur}

Faculté d'Éducation de l'université de Montpellier

\section{Édition imprimée}

Date de publication : 1 décembre 1995

Pagination : 67-82

ISSN : 1167-315X

\section{Référence électronique}

Bernard-Xavier René, "La culture corporelle », Tréma [En ligne], 8 | 1995, mis en ligne le 20 septembre 2013, consulté le 21 avril 2019. URL : http://journals.openedition.org/trema/2141 ; DOI : 10.4000/ trema.2141

Ce document a été généré automatiquement le 21 avril 2019

Trema 


\title{
La culture corporelle
}

\author{
Bernard-Xavier René
}

1 Nous sommes dans la civilisation de la vie. Jamais la vie n'a eu autant de prix. Jamais nous n'avons été si inquiets pour elle, si sensibles à ce qui la menace ni si désireux de l'optimaliser. Peut-être parce que nous n'avons qu'elle. Peut-être parce que nous n'en avons qu'une. Ou, plus simplement, parce que nous pouvons nous payer le luxe de sa qualité. C'est pourquoi, en tous cas, le corps a pris dans notre civilisation et notre existence une importance fabuleuse. Parfois jusqu'à l'excès. Sa présence s'est d'ailleurs d'autant mieux imposée qu'il est plus ubiquiste qu'on peut l'imaginer et plus polymorphe qu'on peut le penser. Jaillir en une multitude de représentations et se répandre en une constellation d'images est, certes, sa façon d'envahir le terrain et de s'imposer au centre de notre vie. Mais cela tient aussi à sa nature même qui est culturelle et mentale.

\section{Le temps des bilans}

2 Les fins de siècle sont propices aux bilans. Quand il s'agit, en outre, d'une fin de millénaire on ne peut plus y échapper. On n'en finit pas de se demander ce qu'on y a fait et ce qui nous a fait ce que nous sommes. Place aux historiens! On s'interroge aussi sur les leçons que l'on pourrait en tirer et sur ce que nous avons appris sur nous-mêmes. Place aux philosophes! Parmi les phénomènes marquants de ce siècle, les optimistes se plaisent à relever un certain nombre de progressions spectaculaires dont l'accélération semble s'être accrue au fur et à mesure que l'on se rapprochait du troisième millénaire : mouvement de démocratisation, montée du sujet et des droits de l'homme, de la tolérance et du respect des différences, efforts d'éducation, bond en avant des sciences humaines, développements technoscientifiques sans précédents, transformation des modes de production, des formes et des moyens de consommation, souci de l'écologie et de l'environnement, essor des loisirs et amplification du temps de non-travail, avec les changements structurels qui peuvent en résulter à terme... 


\section{L'envers du tableau}

3 Cette histoire ne s'est toutefois pas écoulée comme « un long fleuve tranquille ». Elle n'est pas allée sans régressions brutales et sans reculs sauvages qui interdisent définitivement toute vision naïvement optimiste de l'homme et toute conception béatement positiviste du progrès! Les réalistes n'ont guère de peine à montrer que ce siècle des progrès spectaculaires restera aussi comme l'un des plus meurtriers, des plus sanglants et donc des plus inquiétants (ou des plus éclairants) sur ce que l'on peut espérer de l'homme. Deux guerres mondiales, sans compter les autres, le règne du terrorisme, de l'intolérance, des racismes, des fanatismes et des intégrismes, des génocides et des purifications ethniques évoquent plus l'état de jungle que les progrès d'une civilisation! Avant de voir s'écrouler deux formes du totalitarisme : le nazisme et le communisme soviétique, il aura fallu les millions de morts de la seconde guerre mondiale, la Shoa, le goulag et les soixante-dix ans de stalinisme. Chaque décennie, chaque jour apportent encore leur pesant de nouvelles sordides, de boucheries, de morts, de sang et d'exactions insoutenables auxquelles nous sommes d'autant plus réactifs, il est vrai, que nous sommes justement mieux informés et plus sensibles à certaines valeurs.

Cette histoire ne va pas non plus sans revers ni sans poser des problèmes qui sont loin d'être résolus! L'un des revers les plus dramatiques du modernisme progressiste est sans doute le massacre de l'environnement. Le souci de l'écologie n'est pas né du hasard. Mais si son urgence est une chose, sa mise en œuvre en est une autre. Ce siècle productiviste se solde par le gaspillage des ressources, la pollution multiforme, les chèques en blanc sur l'avenir... et son consumérisme semble de moins en moins destiné à satisfaire de vrais besoins qu'à entretenir le productivisme, à accroitre les profits et à affirmer une nouvelle forme de pouvoir. Quant aux problèmes géopolitiques, politiques et sociaux: accroissement du fossé entre pays pauvres et pays riches, inégalités sociales, sociétés duales, tiers monde, quart monde, exclusions, corruptions, etc. ... qui accompagnent cette quête absurde du profit et du pouvoir, ils frisent de plus en plus l'insupportable. Cet envers du tableau a de quoi laisser l'optimiste sceptique, le militant vigilant, l'intellectuel insolent. 


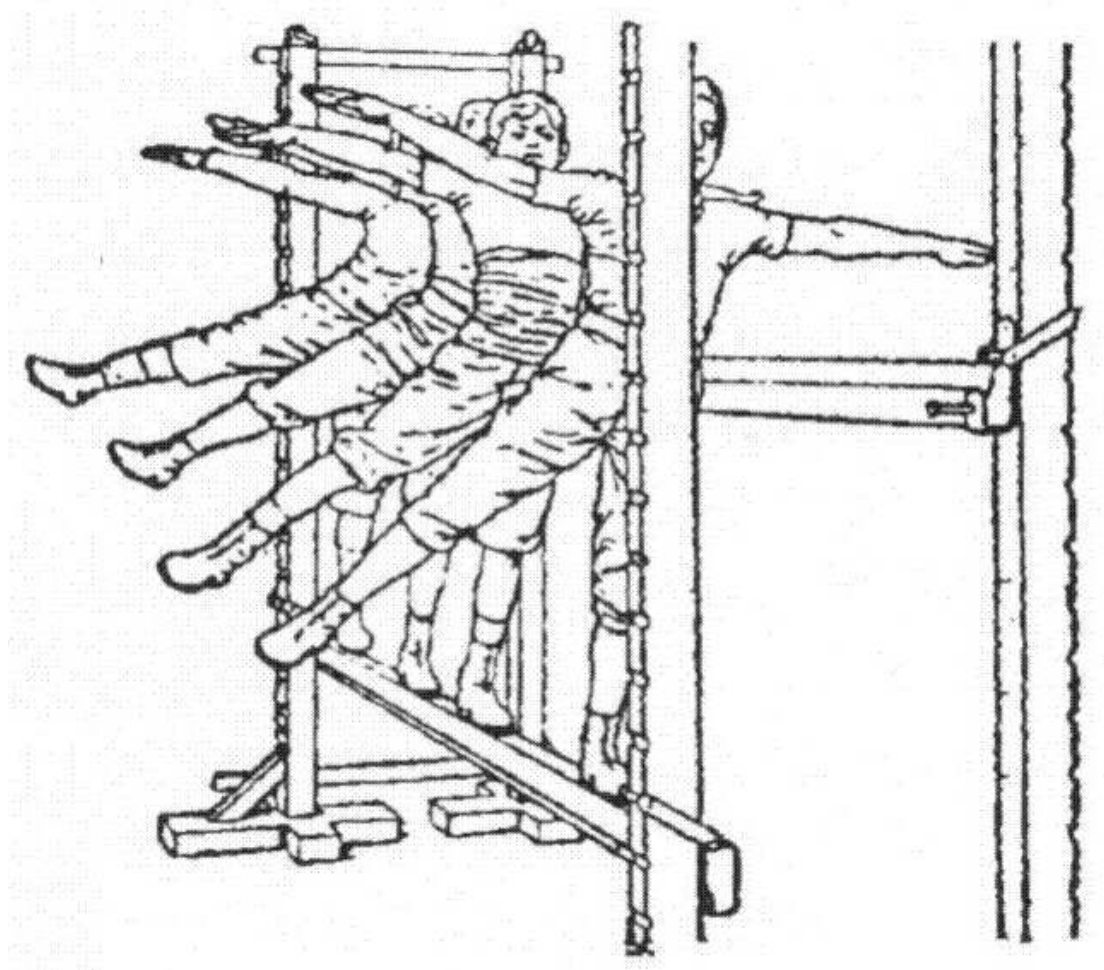

Exercices physiques sur la poutre pour élèves de 9 à 11 ans. Ministère de l'Instruction Publique,

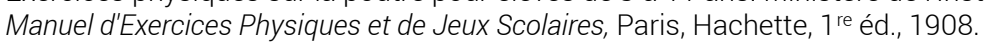

Allons plus loin. Même ce qui se donne pour un progrès incontestable n'est pas insoupçonnable. Car rien, en ce domaine, n'est tout noir ou tout blanc : il faut abandonner la vieille dichotomie manichéenne. Nos grandes conquêtes technoscientifiques, les grands appareils de production, de distribution ou d'énergie qui quadrillent nos sociétés et nos modes de vie posent autant de problèmes qu'ils prétendent en résoudre. Nos systèmes les plus sophistiqués, éducation ou santé par exemple, révèlent chaque jour leurs lacunes, leurs dysfonctionnements voire leur contre-productivité. Sans doute ne s'agit-il pas de "jeter le bébé avec l'eau du bain », comme on dit, et faut-il travailler dans la nuance mais tout ce qui se réclame de l'idéologie du progrès doit être étudié dans ses prétentions, ses finalités et ses conséquences, ses avantages supposés et ses inconvénients cachés. À qui profite-t-il vraiment? Rien ne peut légitimement échapper à l'analyse ni se soustraire à l'interrogation.

\section{Le souci du corps}

6 Or, parmi les phénomènes qui ont marqué le siècle, il faut également compter la majoration exceptionnelle du fait corporel. Non seulement le corps a pris une place sans précédent dans notre civilisation mais, si l'on peut dire, nous avons plus de corps qu'on n'en a jamais eu. Non que nos ancêtres n'aient pas été faits de chair, de sang et d'os, comme nous, mais ils n'ont jamais eu un tel souci du corps. Le corps, autrement dit, prend une place inédite dans l'histoire à l'intérieur d'une large mouvance corporelle, exponentiellement amplifiée, qui le promeut et le rend tel qu'il n'a jamais été. Produit du 
phénomène corporel de notre société, il ne se réduit ni à sa localisation concrète ni à la substance dont il est fait: il s'est essentiellement imposé à partir d'une préoccupation multiforme, indéfiniment ramifiée et amplifiée qui n'a cessé de s'étendre à toutes les couches de la population et de s'augmenter, parfois jusqu'à l'exagération, à travers les générations au fur et à mesure que ce siècle s'est avancé. Avoir un corps, en ce sens, c'est se soucier de son corps, lui donner une importance, une valeur qui dépasse sa matérialité et en métamorphose le sens et l'existence. Car plus on s'en soucie, plus on s'en occupe, plus on le majore et plus on se dote d'un corps que l'on n'avait pas. L'explosion du corporel fabrique du corps virtuel qui devient notre réalité corporelle jusque dans le plus quotidien et le plus intime.

Or ce phénomène corporel n'est pas détachable de la plupart des autres aspects marquants du siècle que nous avons notés. Et, comme eux, il doit donc passer au crible de l'analyse. Non seulement il leur est contemporain mais, d'une certaine façon, ils sont tous, de près ou de loin, en interconnexion avec lui, comme dans tout système complexe dont les éléments sont, plus ou moins directement, interdépendants. Sans revenir sur les aspects les plus noirs qui illustreraient ce propos en négatif et qui suffiraient à faire du corps un enjeu essentiel et un lieu de passage incontournable qui le situe au centre du débat (mort, tortures, emprisonnement, viols, famine, atteintes à la santé, etc.), pratiquement tous les aspects que l'on juge habituellement comme les plus positifs : démocratisation, sujet, droits de l'homme, habeas corpus, consommation, médecine, travail, science, technologie, etc. s'articulent tous, de près ou de loin, au corps, à son bienêtre, ses plaisirs, ses soins, ses loisirs et l'ensemble des sollicitudes et des sollicitations dont il fait l'objet. Autrement dit, ils ne sont pas seulement contemporains : il y a entre eux une relation intime. Le corps ne serait pas ce qu'il est devenu si démocratisation, sujet, consommation, éducation ..., n'avaient pas pris la place qu'ils occupent dans notre civilisation.

8 Sans doute ne s'agit-il pas de tomber dans l'illusion du tout corporel : tout dans notre culture ne se ramène pas ou, plutôt, ne se réduit pas au corporel mais le corps, la vie, y ont pris une ampleur sans précédent et infiltrent la plupart des grands domaines de cette civilisation. L'intérêt dont ils font l'objet circule à travers une profusion de circuits et se cristallise en une multitude de représentations.

\section{Un phénomène complexe}

Cette façon de parler du corps, bien entendu, relève autant de son concept que de son percept. D'ailleurs, ce que l'on en sait et ce que l'on en voit est déjà infiltré par ce que l'on en dit, ce que l'on en sent, ce que l'on en souhaite et par ces myriades d'images du corps qui peuplent notre univers interne et externe, s'imposent à nous et nous apprennent ce qu'il est et ce qu'il doit être. Mixte de nature et de culture, il est donc moins une réalité biophysique qu'un produit sociohistorique complexe pris dans un ensemble de réseaux qui lui donnent une réalité et une fonction qui dépassent de loin sa dimension naturelle. C'est cette complexité que nous vivons sans en avoir une conscience claire mais que peut en révéler une réflexion attentive.

Dans l'instant où au quotidien, toutefois, nous oublions volontiers cette complexité et nous ramenons notre corps à une simplicité de façade, à une image dominante et/ou aux limites de son support perceptible sans regarder plus loin. Sans plus nous interroger sur 
le foisonnement que cache cette apparente simplicité ni sur la multitude d'images que recouvre cette fausse unité, qui infiltre cette perception syncrétique et qui structure nos attitudes et nos comportements avec d'autant plus d'influence que nous n'y prêtons pas attention. Bref, sans bien nous rendre compte que cette réalité familière est le nœud d'un croisement intense de lignes de forces multiples et le point de passage d'une débauche d'aspirations hétérogènes, disparates et divergentes. Mais c'est facile, c'est pratique et ça suffit pour vivre! Car vivre c'est à la fois se souvenir et oublier. L'attention au présent réclame non seulement la puissance cumulative d'une mémoire qui donne de la profondeur à notre expérience et des ressources à notre action mais aussi une capacité d'oubli qui nous permet de ne retenir que ce dont nous avons besoin pour nous ajuster de façon pertinente au présent. Vivre, c'est ainsi garder en nous cette diversité pléthorique dont seul tel ou tel aspect peut ponctuellement prévaloir et devenir notre réalité corporelle pour une durée indéterminée, très brève ou très longue, selon les coïncidences des circonstances et de nos évolutions personnelles; et c'est aussi, en même temps, oublier cette diversité au profit de telle image dominante plus ou moins éphémère.

11 Pourquoi faire simple, en effet, quand on devrait faire compliqué? On peut, au moins repérer facilement deux types de raisons. Les unes plus psychologiques, les autres d'ordre socio-historique. Psychologiquement, c'est une question d'économie. Il est moins coûteux et plus efficace, cognitivement, de nous en tenir à ce qui s'impose dans le présent, à ce qui se donne dans l'immédiat, à ce que nous voyons ou à ce que nous croyons que de faire un effort d'analyse souvent inutile pour les besoins de la vie courante. L'image évite la réflexion, l'élément est pris pour l'ensemble, l'opinion tient lieu de certitude. Les raisons cognitives et individuelles que l'on peut évoquer sont, bien sûr, les plus évidentes. Elles ne doivent pas en faire oublier d'autres qui sont liées aux situations historiques particulières. Que notre civilisation ait été peu à peu transformée par l'amplification fabuleuse de la vague corporelle qui la soulève et qu'elle produit, n'empêche pas que telle époque historique ait pu opérer des choix, valoriser telle image du corps plutôt que telle autre et contribuer ainsi, sans bien toujours s'en rendre compte, à l'extension de l'éventail des modes corporelles. Une situation historique ou institutionnelle donnée, avec ses contraintes et ses aspirations, peut opérer des mises en perspective, privilégier certains points de vue, avantager certains aspects partiels et donc certaines images. $\mathrm{Ce}$ qu'elle dit et montre du corps Justifie ce qu'elle en fait. Ce qu'elle en fait sert ses valeurs et ses finalités. 
"Le développement musculaire chez les jeunes filles. Type remarquable de développement musculaire en finesse. Pose faisant particulièrement ressortir le modelé des muscles de la région dorsale et lombaire, formant le sillon de la colonne vertébrale ».

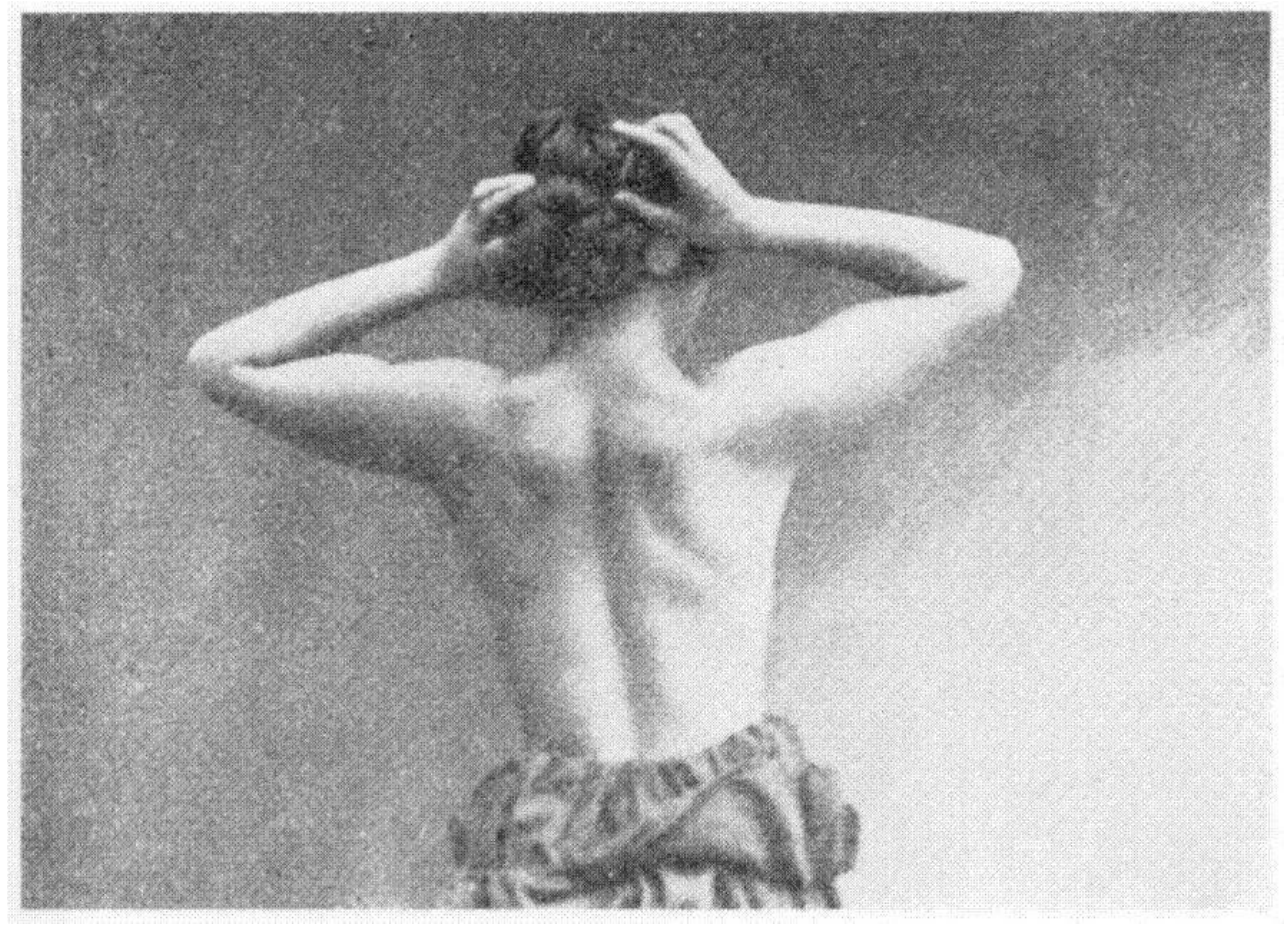

Georges Hébert, L'Éducation Physique Virile et Morale par la Méthode Naturelle, Tome I, Paris, Vuibert, 1936.

Mais, économie psychologique ou stratégie historique, une image du corps ne peut en cacher la prolifération. Ce qui, d'ailleurs, dans le court terme, peut être considéré comme une opération réductionniste, parfois très sévère, liée à des stratégies circonstancielles ou à des réactions régressives, peut se révéler, sur le long terme, comme une facette de plus de la panoplie du corporel. Ne serait-ce que parce qu'il déclenche, fut-ce par opposition, des aspirations nouvelles et contradictoires ou parce qu'il ajoute ce qui n'est que la réalité d'un moment à toutes les autres qui l'ont précédé et à toutes celles, innombrables, qui suivront et que nous n'avons pas fini de vivre ni d'inventer aussi bien au niveau historique qu'individuel. Sans cloute le réductionnisme doit-il toujours être interrogé. Psychologiquement, il s'apparente au pathologique; historiquement, il est souvent suspecté de masquer sous son caractère partiel et mutilant la partialité d'un système de valeurs tendancieuses. Mais, concernant le corps, il apparaît aussi, rétrospectivement, que les épisodes historiques qui sont réputés l'avoir le plus opprimé sont aussi ceux qui lui ont consacré un maximum d'attention et de curiosité, ne serait-ce que pour le circonscrire et en fouiller les aspects les plus secrets et les plus intimes, et qui ont peutêtre, en fin de compte et contre toute attente, le plus contribué à son irrésistible ascension. Regardons-y d'ailleurs à deux fois. On a dit le corps réprimé, étouffé par les siècles de moralisme, d'interdits et de prescriptions. Mais n'était-ce pas un prétexte habile pour réclamer sa libération, légitimer son affirmation, justifier la revendication de suppléments de corps?

13 Autrement dit, si le recours à un corps simple qui répond aux besoins d'économie du cognitif n'efface pas la luxuriance du corporel, la focalisation historique sur une 
représentation privilégiée du corps ne freine pas non plus son extension et même, au contraire, peut y contribuer ou servir, en tous cas, d'alibi rétrospectif à son inflation.

\section{La multiplicité du corps}

14 réalité en soi, la même toujours et chez tous. C'est une réalité virtuelle à géométrie variable, multiple et mouvante qui change avec les époques, les points de vue, les modes, les attentes, les milieux, les personnes et les enjeux. Que l'on aborde la question du point de vue de la sociohistoire du phénomène corporel ou du point de vue plus individuel des sujets corporels, on a affaire à la même pluralité fluctuante et à la même invasion irrépressible. Quelques développements suffisent à s'en faire une idée plus précise encore. connaissons semble bien résulter, en effet, d'une agglutination progressive et désordonnée d'intérêts pour le corps qui en esquissent peu à peu les contours, en majorent les aspects et en augmentent les domaines d'extension. Ils se font jour graduellement, à travers les siècles, à partir de points multiples, pour des raisons diverses, sans plan d'ensemble préétabli ni finalité commune préconçue, des confins du moyen âge et de la renaissance où il prend son essor jusqu'à notre siècle où il enfle démesurément et s'étale dans toutes les directions. Pour des raisons qui leurs sont propres, juristes, religieux, médecins, pédagogues, militaires, professionnels, experts de toutes corporations et spécialistes de toutes disciplines prennent le corps pour objet de leurs études et/ou de leurs pratiques. Leurs activités qui se développent d'abord de façon spécifique, s'expansent, rencontrent des similitudes transversales, des connivences interdisciplinaires, des analogies transdisciplinaires et finissent par converger, se rassembler et coexister, dans cet amas confus, cet agrégat composite mais de plus en plus riche qu'est la vogue du corps. Elles se juxtaposent, s'amalgament puis s'articulent jusqu'à ce qu'un phénomène d'ensemble paraisse en naître et qu'à leur croisement se dessine, se conforte et grossisse un effet boule de neige, fruit du hasard historique et des nécessités individuelles ou corporatistes. Cet effet qui fait de plus en plus d'effet engendre un effet d'être: le fait corporel, aussi puissant qu'une avalanche et aussi diversifié que ses origines.

Il est multiréférentiel par ses lectures. Cette différenciation du corporel s'appuie et s'enrichit historiquement de la multiplicité des lectures qui en sont faites par les différentes spécialités qui s'y intéressent et s'en emparent. Autant de points de vue, de méthodes, d'objectifs, autant de façon de penser et de traiter le corps. Les excellents travaux de Michel Bernard ${ }^{1}$ ou de Jean-Marie Brohm ${ }^{2}$ suffisent, sans que l'on y revienne, à montrer combien le corps est pulvérisé en une multitude de représentations : autant dedisciplines, autant d'auteurs, autant d'évolutions dans leur pensée... autant d'images ou de concepts. Autrement dit, c'est moins le corps qui est ou qui n'est pas multiple que les regards, les discours et les traitements qui en font un objet de recherche, de réflexion ou de pratique et qui, premièrement, le constituent en objet et, deuxièmement, en font un objet multiple. Ce que nous en disons et ce que nous en faisons le promeut comme réalité et l'installe dans le mouvement de la vérité. Le corps est moins riche d'une multidimensionnalité intrinsèque qu'il ne le devient historiquement par nos pratiques discursives et non discursives. En un mot, le corps qui nous paraît la chose du monde la 
plus naturelle et la mieux partagée est un pur produit. Produit de l'histoire et produit des hommes qui la font autant qu'elle les fait.

Il est différencié par son histoire car non seulement ses sources sont multiples et multiréférentielles mais elles ne jaillissent pas toutes en même temps, spontanément, au tournant d'une date historique et elles ne conduisent pas du tout à une réalité unique et monolithique. Elles apparaissent progressivement, se construisent et se modifient graduellement, se juxtaposent, se superposent et s'opposent, s'articulent ou se contaminent les unes les autres dans chaque discipline ou chaque lieu d'émergence avec d'éventuelles interactions entre ces espaces de production. On aboutit ainsi à un ensemble volumineux mais disparate dans le détail et sans véritable cohérence fait de filiations ou d'oppositions de concepts, de successions d'images, de parallélismes de représentations, de convergences ponctuelles ou de divergences profondes. Les modèles $\mathrm{du}$ corps, juridiques, pédagogiques, physiques, biomécaniques, anatomiques, physiologiques, neurologiques, philosophiques, psychologiques, psychanalytiques, didactiques, etc. se suivent sans se ressembler ou coexistent plus ou moins pacifiquement. Ils se remplacent mais sans se supprimer totalement et se déposent en strates successives où ils continuent à persister et à avoir une influence plus ou moins profonde suivant les moments, les groupes et les circonstances.

18 Il est omniprésent par sa variété. Le fond commun dans lequel converge sa multiplicité s'organise, en effet, en un réseau extraordinairement ramifié, fait d'articulations multiples, de regards, d'observations, de savoirs, d'attentes, de services, de normes, de règles, de prescriptions, d'aides, de sollicitations, de sollicitudes, d'éducation, de conditionnements, de mises en forme, de productions et de consommations, etc. La puissance du phénomène s'appuie précisément sur cette mosaïque différenciée qui lui permet de «ratisser large» et de ne laisser personne indifférent ni hors circuit. Au corporel on ne peut échapper. Sa présence et ses images peuplent notre environnement culturel et économique, fleurissent dans nos médias, de l'affiche au magazine et à l'écran, de la revue scientifique au Minitel rose, s'étalent dans la publicité, s'infiltrent dans tous les interstices et s'immiscent dans toutes les circonstances. Non seulement chacun peut trouver, dans l'hypermarché des images du corps celles qui lui convient le mieux et en changer à volonté s'il le souhaite, mais toutes les réclamations, toutes les revendications toutes les contestations se font à l'intérieur du phénomène global et sous forme de surenchères corporelles et d'exigences de suppléments de corps. Nous voulons toujours plus de corps. Et sans doute l'immense sollicitude que nous lui témoignons n'est-elle que notre nouvelle manière de nous aimer nous-mêmes puisque notre corps c'est nous. Il a pris, en cela, la place de l'âme. Si bien que, plus l'on va et plus on en a. Plus nous sommes corporels. Plus nous sommes emportés par la déferlante corporelle et la frénésie de la consommation de corps. Corps et consommation ne sont d'ailleurs pas aisément séparables. L'important n'est pas d'être pour ou contre telle représentation, telle pratique, tel usage, telle mode ou tel modèle. L'important c'est d'en être, de trouver sa place, de participer et de consommer. De faire partie de ce dispositif global qui s'est peu à peu installé et qui capte nos intérêts, nos attentions, nos curiosités et nos goûts et qui fait ses profits de la consommation de corporel. Fort de son omniprésence qui imprègne le corps social et en imbibe le tissu en profondeur, il impose son omnipotence d'une manière de plus en plus discrète et silencieuse mais non moins réelle et efficace. 
"Le maintien de l'équilibre. Monter à une échelle avec les jambes seulement, les bras servant de balancier pour maintenir l'équilibre. Le maintien de l'équilibre nécessite un effort mental soutenu qui fatigue rapidement $»$.

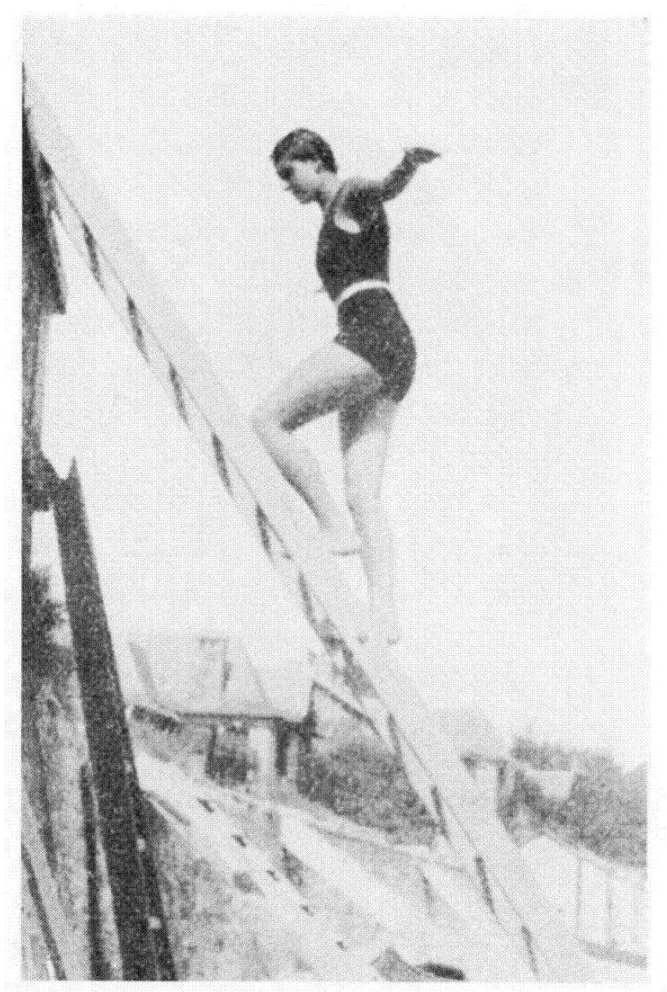

Georges Hébert, L'Éducation Physique Virile et Morale par la Méthode Naturelle, Tome I, Paris, Vuibert, 1936.

19 Il est omnipotent par son ampleur. Après une explosion assez fracassante faite d'effraction provocatrice, de libération effervescente et d'hédonisme sans réserve dans les années 70 et 80 , la révolution corporelle semble s'être calmement étalée dans le confort d'une quotidienneté dont elle est le point de passage obligé et la préoccupation première ${ }^{3}$. Le corps roi s'est banalisé en se diluant dans nos habitudes, car non seulement il fait partie du paysage mais il est notre constant horizon. Notre accoutumance ne fait que témoigner de son installation dans nos structures perceptives et mentales. Il va tellement de soi que l'on en vient à se demander comment il pourrait en être autrement! On peut même aller jusqu'à dire, sans crainte d'exagérer, que, aujourd'hui, le nouveau lien social s'organise autour de ce souci du corps, tant ce souci collectif, généralisé par son extrême diversité, anime, aimante et rassemble le corps social, tant il polarise nos activités, nos valeurs et nos finalités. Cette mouvance corporelle sert ainsi de support à la dynamique d'une nouvelle forme de pouvoir, soft et positif, qui gère la vie et que Foucault appelait le "biopouvoir $»^{4}$. Elle trouve son représentant dans le corps, point de rencontre et de cristallisation de ses attentions, dans ses besoins, sa santé, son bien-être, ses plaisirs, ses loisirs, son confort, sa séduction, sa sexualité, son intimité, ses usages, ses pratiques, ses performances, ses modes et ses modèles.

Il est hétérogène par culture. Cette hétérogénéité serait vérifiable à travers l'histoire et le temps des civilisations ou d'une même civilisation. Elle pourrait faire également l'objet d'une ethnologie comparative qui se pencherait sur les usages, les techniques du corps et le rapport au corps d'une société à une autre, à la même époque. Mais elle est également 
vraie, à l'intérieur d'une même société, comme la nôtre, d'un groupe social à l'autre. Sans revenir sur ce que nous avons dit, sur la cohue et la coexistence des images, des pratiques et des représentations et des consommations, d'une part, et sur l'émergence éphémère de telle mode ou de tel modèle provisoirement distingué, d'autre part, on peut se reporter plus particulièrement aux travaux de sociologie, dans la ligne de Pierre Bourdieu, pour voir comment se dessinent et se distribuent chez les individus des régularités, des tendances et des habitus corporels selon les espaces sociaux d'origine. Travailleur de force, col blanc, grand bourgeois, ajoutons même : homme ou femme, n'ont pas le même rapport au corps. Ils ne le voient, ne le pensent, ne le traitent ni ne le vivent de la même manière. Ils ne partagent ni les mêmes goûts, ni les mêmes pratiques, ils n'ont ni les mêmes soins ni les mêmes intérêts ni les mêmes usages du corps. Bref, ils n'ont pas le même corps. Sociologie du goût et approches sociologiques des pratiques corporelles et sportives, en particulier, ont largement contribué à nous débarrasser de l'illusoire homogénéité du corps. L'idée selon laquelle quand le roi est nu il est un homme comme les autres parce qu'il a le même corps est une idée fausse. Ni dans leur lit ni dans leur salle de bain le cadre supérieur et l'ouvrier spécialisé n'ont le même rapport au corps. $\mathrm{Ne}$ parlons pas des marquages corporels dus à la forme de travail, de leur art de la table ou du vêtement, des choix sportifs ou esthétiques, de l'aisance et du maintien voire de la sexualité. Partout la distinction s'impose.

21 Il est polymorphe par son vécu. Au-delà de l'histoire, de l'ethnologie, de la macro et de la microsociologie, l'étude des individus confirme, par son entrée spécifique, cette multiplicité du corporel. La différence y est à la fois interpersonnelle et intrapersonnelle car non seulement nous n'avons pas le même corps mais surtout nous ne vivons pas de la même façon. La différence qui saute aux yeux est évidemment celle des caractéristiques physiques: elles nous permettent de nous reconnaître et de nous identifier. Notons toutefois qu'elles ne sont pas aussi neutres que le descriptif de notre carte d'identité. La couleur de la peau, des yeux ou des cheveux, la stature ou le poids ne disent rien à eux seuls de l'agrément d'un visage, de la vivacité d'un regard, de l'énergie dans l'action, de l'aisance des attitudes ou du charme d'une voix. C'est dans cette rencontre et cette combinaison unique et singulière de multiples caractéristiques qu'une personne s'offre au regard des autres mais aussi au sien. Or si chacun peut apprécier une personne et son physique à partir de son point de vue propre, la partie est plus difficile à démêler quand il s'agit de soi : se croisent et se combinent, en effet, en notre jugement l'image que les autres nous renvoient de nous et notre double perception de nous-mêmes, à la fois externe et interne sans que l'on puisse toujours bien distinguer d'une façon péremptoire et définitive le poids de l'une et des autres. Rien de moins stable et de plus incertain que la façon dont nous vivons notre corps, dont nous nous apprécions, nous voyons, nous aimons, nous supportons ou nous détestons. Ce qu'est notre corps pour nous varie selon le moment, l'humeur, l'état de santé, l'âge, la rencontre. Suivant que nous sommes en forme, frais et dispos ou fatigué et déprimé. Bref, en fonction des multiples aléas et retournements de notre vie biologique et psychologique. A la limite, peu importent notre réalité corporelle, notre âge, notre esthétique et même notre santé. L'important c'est ce que nous en pensons, la façon dont nous le sentons et le ressentons, tant il est vrai que, profondément, le corps c'est dans la tête 5 . Les différences ne sont donc pas seulement objectives ou quantifiables, d'une personne à l'autre ou d'un âge à l'autre chez un même individu : elles sont encore 


\section{Le corps comme représentation} du médecin à l'éducateur. En Education Physique, par exemple, comment juger à la même aune dans les activités physiques qui leur sont imposées, quand il s'agit de courir ou de sauter par exemple, des élèves de titille ou de poids différent? Comment juger ceux qui ont grandi trop vite et ceux qui ne maîtrisent pas bien un schéma corporel en pleine reconstruction, ceux qui sont pleins d'énergie et ceux qui se traînent dans un corps lourd ou mou, ceux qui sont bien dans leur corps et ceux qui le vivent mal? Quels racismes latents, quelles humiliations plus ou moins volontaires attendent l'obèse, le maladroit, le lymphatique? Quel rapport à leur corps, quelle image d'eux-mêmes y construiront-ils? Cette difficulté est d'autant plus difficile à accepter et à gérer par les enseignants d'EPS qu'ils ont eux-mêmes, en général, un rapport positif et réussi à leur corps et qu'ils ont du mal à imaginer qu'il peut en être autrement pour d'autres. Ce n'est, parfois, qu'avec le poids des années et les effets négatifs à long terme d'une activité physique trop intense dans la jeunesse, qu'ils commencent à se rendre compte que le corps n'est plus ce qu'il était et qu'il n'est pas ce qu'ils croyaient quand ils se pensaient encore incassables et immortels, qu'ils débordaient de force, de vigueur, d'insouciance et d'impétuosité. Alors les différences s'accusent et peuvent peut-être mieux se comprendre...

\section{Le corps comme représentation}

Le corps est donc le produit d'une double représentation : représentation sociohistorique et représentation psychologique. Ce qu'est le corps pour une époque donnée, un groupe social donné voire un individu précis est fonction de ce qu'ils sont, de leurs caractéristiques propres, des tensions qui les animent, de leurs préoccupations essentielles, de leurs attentes, de leurs espoirs. 

de pouvoir qui s'y est développée. Même s'il parait se trouver au centre des attentions ce n'est pas uniquement pour lui-même qu'il est visé mais plutôt en tant que lieu de passage, moyen pour d'autres fins. Ce qu'est le corps, en ce sens, est toujours relatif aux enjeux dont il est l'objet, aux ambitions qui le traversent, aux projets dont il est le support et qui le constituent, au moins provisoirement, en le cristallisant dans une représentation dominante ou en le pulvérisant en une multitude d'images et de concepts. Produit à l'entrecroisement des multiples forces qui l'engendrent et des maillages très ramifiés qui le profilent, il évolue avec les fluctuations sociohistoriques dont il est l'effet. Cet effet sociohistorique est plus large que sa représentation concrète et bien moins nettement délimité. Cette représentation n'en est, d'ailleurs, qu'une parmi d'autres, ni plus neutre ni plus innocente qu'une autre, et elle appartient à ce magma fluctuant qu'est le corps pour nous. c'est nous-mêmes qui sommes en jeu, nos sensations, nos émois, nos désirs, nos plaisirs, nos forces et nos faiblesses, nos contradictions. Et, à travers le rapport existentiel privilégié que nous avons avec notre corps, nous pouvons juger de l'instabilité de sa représentation. Ce corps qui est nous n'est pas, en effet, un corps en soi. Il est un corps pour nous. Il relève de notre représentation car de la représentation nous sommes et nous ne pouvons pas sortir. Derrière nos images et nos concepts du corps nous ne pouvons trouver que d'autres images et d'autres concepts du corps, sans image dernière, sans espoir de synthèse ni point de vue de Sirius. La façon dont nous vivons notre corps, pourtant si immédiate, ne peut tirer aucun privilège de cette immédiateté. Elle n'est qu'une façon parmi d'autres, souvent très précaire et très passagère d'ailleurs, qui peut trouver sa place clans la totalité ouverte et indéfiniment extensive des manières de vivre son corps et de se représenter le corps. Sans doute y a-t-il une dialectique très étroite entre notre vécu corporel et nos représentations : c'est pourquoi la fluidité très mobile de ce vécu s'exprime en une abondance d'images divergentes et en une conception très plurielle du corporel. Mais cette diversité et cette conception plurielle prend elle-même place dans l'ensemble ouvert des représentations du corps. Autrement dit, quelle que soit votre conception, même la plus étroite, la plus unidimensionnelle et la plus unitaire, elle vient gonfler le flot des représentations et donner raison à la diversité. Elle vient justifier la conception plurielle du corps.

Mais encore faut-il le savoir et le comprendre pour saisir quelles conséquences on peut en tirer. Nous sommes, en effet, des êtres du symbole et nos représentations règlent nos rapports aux choses: elles guident ce que nous faisons et influent sur ce que nous devenons. Or la façon dont nous nous représentons notre corps influe en retour sur la façon dont nous le vivons et sur ce que nous en faisons, sur notre manière de le traiter, et quand on se trouve en responsabilité du corps des autres, sur notre façon de leur apprendre leur corps et de les positionner par rapport au phénomène corporel.

\section{L'apprentissage du corps}

29 Nous apprenons, en effet, notre corps non seulement à travers notre expérience propre mais aussi à partir de la médiation des autres, de notre milieu, de notre époque. Les situations qu'ils nous préparent, les pratiques qu'ils nous concoctent, les envies qu'ils incitent, les attitudes qu'ils valorisent, les plaisirs qu'ils excitent, les usages qu'ils 
prescrivent, encadrent et contrôlent, les images dont ils nous environnent, les représentations qu'ils distillent tout au long de notre éducation, etc., influent sur notre façon de le vivre et de le penser, sur notre façon d'être corporel et d'avoir un corps. motrice et d'un savoir neuromusculaire.

D'autre part, et à propos du premier, peut se construire un apprentissage par corps ${ }^{6}$. Si la médiation est suffisante et la pédagogie bien conduite, à travers notre expérience corporelle, nos engagements physiques dans un milieu, nos activités motrices en situation, la totalité de la personne sensible, affective, cognitive et conative, est sollicitée, invitée à se mobiliser et à développer ses savoirs et ses savoir-faire, ses opérations mentales et ses équilibres personnels et relationnels. L'effort du pédagogue, au lieu de se contenter de viser l'acquisition d'un geste, le montage d'une habileté ou la réussite d'une performance, aide à transcender l'immédiat de la situation pour en tirer ce qui est le plus riche pour l'élève, pour comprendre ce qu'il fait, comment il le fait et pourquoi il le fait. Ce qu'il a voulu lui faire acquérir, sentir. Ce à quoi il a voulu le sensibiliser. Il conduit « l'élève » à « s'élever » au-dessus de ce qu'il vient de faire pour en tirer les leçons, pour préparer les situations à venir dans la discipline, certes, mais aussi pour d'autres situations scolaires et non scolaires. Pour sa vie. Pour qu'il s'habitue à donner du sens à ce qu'il fait. À prendre du pouvoir sur sa vie. Qu'apprenons-nous à travers ce que nous faisons? Quels transferts préparons-nous? Et, plus simplement, nous préparons-nous, nous habituons-nous, à transférer? À nous enrichir de notre expérience? 
"La défense par la lutte. Jeunes filles exécutant une lutte élémentaire de traction par la nuque. Le cou est souvent inesthétique chez les jeunes filles, à cause de sa gracilité. La lutte permet de développer ses attaches et de lui donner sa forme normale».

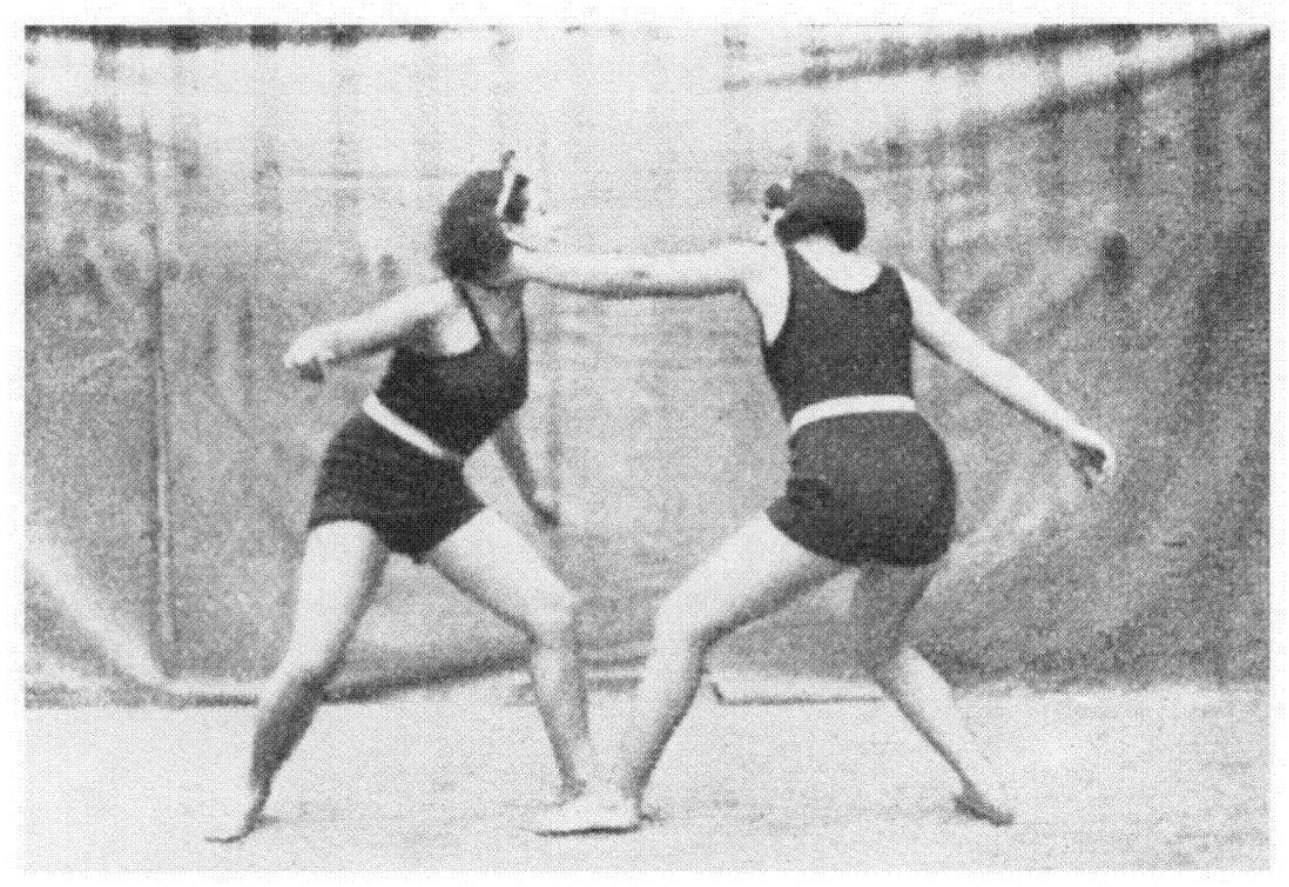

Georges Hébert, L'Éducation Physique Virile et Morale par la Méthode Naturelle, Tome I, Paris, Vuibert, 1936.

Ce peut être, enfin et surtout, le moyen d'une véritable éducation où l'on apprend à se situer par rapport au corporel d'une manière active et critique. Nous vivons, en effet, dans une culture du corps où il faut apprendre et comprendre la diversité du corporel. La connaître, la reconnaître et la respecter en soi et chez les autres ${ }^{8}$. Il faut savoir s'ouvrir aux différences du corps. Apprécier les différentes modalités de l'expérience corporelle. L'ouverture récente mais encore insuffisante de l'Éducation Physique à différents registres de pratiques semble, de ce point de vue, correspondre à un pas en avant. Mais, à côté de cette incontournable pluralité, il importe aussi d'apprendre à relativiser le corporel pour éviter ses pièges et ses excès. Le relativiser personnellement, car si nous nous y réalisons il n'est pas non plus le tout de la personne ni des manières de s'épanouir. Et le relativiser culturellement, car l'inflation du corps doit nous mettre en garde à la fois contre son exploitation dans les circuits économiques ou sportifs, contre les vertiges de la "défonce » qui gangrènent les populations fragiles et contre son utilisation par le processus politique comme moyen de neutralisation et d'anesthésie ou de contrôle et de normalisation silencieuse.

La sensibilité aux excès soulève, tout particulièrement, quelques questions actuelles et urgentes. Elle pose, notamment le délicat problème du plaisir qui court en filigrane derrière toute cette saga du corporel. L'inflation du corporel joue, en effet, sur la course au plaisir et sur la spirale de sa recherche de plus en plus effrénée. Sans même s'aventurer dans une problématique morale ou éthique, on peut avancer avec certitude, même du simple point de vue physiologique, que l'homme est né pour le plaisir ${ }^{9}$ et que, dans la grande diversité et instabilité du «toujours plus » corporel, peuvent s'installer des besoins de sensations de plus en plus fortes qui se caractérisent par un seuil de 
tolérance à l'absence d'excitation de plus en plus bas. La moindre minute sans sensation ni stimulation devient insupportable. Il faut s'éclater ${ }^{10}$. Se défoncer. Vivre "off limits». Jouir de l'extrême. Dans la consommation, la performance, l'exploit ou les drogues. Il faut toujours plus de corps et de plaisir, aller vers un plaisir de plus en plus violent au risque de basculer de la violence du plaisir dans le plaisir de la violence. L'état de manque est douloureux. On en arrive rapidement à une sorte de pathologie, qui va de formes encore bénignes et relativement vivables à des formes de conduites compulsives et à une frénésie qui perd le sens de l'utile. Le plaisir bien régulé, en effet, se situe dans une oscillation entre un processus d'activation et un frein. Lorsque l'équilibre est dépassé, un ressort se brise, un cercle vicieux s'installe et il n'y a plus de mécanisme de satiété. C'est l'emballement. Comment ne pas poser ici la question d'une prise de conscience des dérives actuelles et d'une éducation des choix, de la prise de repères, de la mesure et de la gestion réfléchie du corps? Faut-il attendre que se posent des problèmes ultimes de drogue, de "défonce » ou de violence pour s'en occuper, ou savoir très tôt mettre en garde contre l'engrenage, le repérer dès ses formes les plus apparemment inoffensives? Entre : "plus vite, plus haut, plus fort ", et « tout et tout de suite ", il y a une progression logique qui va du comparatif au superlatif. Elle s'oppose à une installation dans la durée. Apprendre à gérer son corps, c'est apprendre à faire et à conduire des choix, sur le long terme, à savoir dire non et à doser. Mais où et quand l'apprend-t-on, dans quelle école, dans quel cours d'Éducation Physique ? Elle conduit également à repenser la notion de santé. La santé n'est ni l'absence de maladie ni le contraire de la maladie. Si on peut l'assimiler à une dynamique de dépassement ce n'est pas pour la ramener frauduleusement au dépassement de soi ou des autres et pouvoir dire « le sport, c'est la santé ", mais pour y voir, l'art d'échapper aux pièges et aux excès, y-compris ceux du sport, l'art de gérer habilement tout ce qui nous écarte de notre équilibre intérieur. On peut être corporellement faible, déficient, handicapé, et être en parfaite santé si l'on a trouvé un équilibre et une harmonie. A l'inverse, on peut être en excellente santé... et néanmoins très malade. Dans cette problématique se joue la véritable responsabilité des spécialistes du corps et des éducateurs d'aujourd'hui.

\section{NOTES}

1. BERNARD M., Le Corps, Éditions Universitaires, 1972.

2. BROHM J. M., «Philosophies du corps : quel corps?» In Encyclopédie Philosophique Universelle, Paris. P.U.F, 1989.

3. cf. RENÉ B. X., "Après le sport pour tous : le corps pour tous ", in Actes du Colloque Anthropologie du sport. Perspectives critiques. Paris. ANDSHA, Matrice. Quel Corps ?, 1991.

4. FOUCAULT M., La volonté de savoir. Histoire de la sexualité T. 1. Paris. Gallimard, 1976.

5. cf. RENÉ B.X., «Le corps c'est dans la tête », in Cahiers Pédagogiques, n² 262, Mars 1988, Paris, CRAP.

6. cf. RENÉ B.X., « Savoir par corps.. in Cahiers Pédagogiques, $\mathrm{n}^{\circ}$ 288. Paris. CRAP. Novembre 1990. 
7. cf. RENÉ B.X., « Pour ne plus apprendre idiot : fondements et modèles d'une pédagogie médiationnelle ». in Bui-Xuân G., Gleyse |. Enseigner l'Éducation Physique et Sportive, Clermont-Ferrand, AFRAPS, 1993.

8. cf. RENÉ B.X., EPS : «L'autre différence », in Différencier la Pédagogie en EPS. Paris, Edit. EPS 1989.

9. cf. VINCENT J.D., Biologie des passions. Paris. Odile Jacob. 1986.

10. cf. RENÉ B.X., «L'équilibrisme », in Pratiques Corporelles, $n^{\circ} 76,1987$; et « Le plaisir du vide », in Pratiques Corporelles, ${ }^{\circ} 79,1988$.

\section{RÉSUMÉS}

L'auteur effectue une analyse philosophique ou anthropologique du phénomène corporel au cours des dernières décennies qui permet d'en percevoir la multiplicité et l'hypertrophie. Ce texte cherche essentiellement à récuser un certain nombre de lieux-communs à re-visiter et rediscuter des stéréotypes et des fausses évidences concernant une prétendue culture corporelle. Le corps est finalement et essentiellement analysé comme un phénomène complexe. Demeure alors une dernière interrogation, celle qui scrute la notion de plaisir corporel. Le passage dans ce domaine du "plus vite plus haut plus fort» au «tout, tout de suite» doit notamment être analysée et critiquée.

The author conducts a philosophical or anthropological analysis of the phenomenon of the body over the course of the last few decades which makes it possible to perceive its multifariousness and extreme expansion. Essentially, this article endeavors to refute a certain number of clichés as well as to pass under review and discuss anew, stereotypes and false obvious truths concerning a so called culture of the body. The body is here in the final analysis essentially analyzed as a complex phenomenon. There remains thereby a final question raised, namely that which closely examines the concept of bodily pleasure. Especially, the evolution in this field, from the principle of "faster, higher, stronger" to that of "everything right away", must be analyzed and critiqued.

\section{INDEX}

Mots-clés : complexité, corps, culture, multiréférencialité, plaisir

Keywords : Body, Complexity, Pleasure, The multidimensional nature of (...]

\section{AUTEUR}

\section{BERNARD-XAVIER RENÉ}

Maître de conférence, CRUISE, Université de Poitiers 